\title{
GRANULOCYTIC EXPRESSION OF CD11B/CD18 AND THROMBOTIC RISK IN PATIENTS WITH MYELOPROLIFERATIVE NEOPLASMS
}

\section{Doroteya K. Todorieva- \\ Todorova, \\ Katya S. Kovacheva ${ }^{1}$, Nikolay T. Tzvetkov, Svetla O. Blazheva ${ }^{2}$, Tzvetan H. Lukanov ${ }^{2}$}

\author{
Hematology Division, \\ Medical University - Pleven \\ ${ }^{1}$ Medical Genetics Section, \\ Medical University - Pleven \\ ${ }^{2}$ Laboratory of Clinical Immunology, \\ Dr. Georgi Stranski University \\ Hospital - Pleven
}

\begin{abstract}
Summary
Myeloproliferative neoplasms (MPN) are clonal hematological conditions characterized by excessive production of one or more cell lines in the bone marrow. The blood cells produced are often hyperactive in their functions, which could lead to complications in the disorder's clinical course. We aimed to define the role of granulocytic CD11b/CD18 expression for the thrombotic risk in MPN patients. We investigated 110 patients with a histologically confirmed diagnosis of a myeloproliferative disease and a control group of 46 healthy volunteers. In the patient group, we found an average expression 4.59 times higher than in the control group. The highest expression was found in a subgroup of patients with polycythemia vera $71.55 \%$ of the patients' neutrophils. In each subgroup with essential thrombocythemia, myelofibrosis, and chronic myeloid leukemia, the patients with a history of thrombotic complication had a higher expression than the patients without such complications.
\end{abstract}

Keywords: CD11b/CD18 granulocytic expression, myeloproliferative neoplasms, thrombotic complications

\section{Introduction}

Myeloproliferative neoplasms (MPN) are hematological diseases with clonal hematopoiesis and increased cell line proliferation. Classical MPNs include chronic myeloid leukemia (CML), polycythemia vera (PV), essential thrombocythemia (ET), and myelofibrosis (MF). These can be divided into two main groups, depending on the presence of Philadelphia chromosome $\mathrm{t}(9 ; 22)(\mathrm{q} 34 ; \mathrm{q} 11)$ - Philadelphia-positive CML, and the rest of the Philadelphia-negative diseases. A point mutation V617F ( $\mathrm{G}->\mathrm{T}$, leading to dispositioning of phenylalanine with valine on the 617 position) of the JAK2 gene (encoding Janus kinase) is typical for the second group. Its prevalence is about $95 \%$ in $\mathrm{PV}$ and about $50-60 \%$ in ET and MF [1-9]. Mutation can be found in hematopoietic cells, leading to excessive cell proliferation and survival benefit by activating Janus kinase and increasing signal transduction in the JAKSTAT signal pathway [8]. Often, MPNs are associated with a higher thrombotic risk with multifactorial genesis: patient-related factors, genetic factors, abnormal coagulation, blood cell variations, and 
cell dysfunction [10-16]. After the year 2000, some studies focused on the role of activated leukocytes in patients with MPN as a thrombotic risk factor, the worse clinical manifestation of the disease, and frequent complications [17, 18]. CD11b/CD18 (Mac-1 complex) expression on the granulocytic membrane is a marker for such activation.

Given these data, we aimed to study the role of granulocytic activation by CD $11 \mathrm{~b} / \mathrm{CD} 18$ expression in MPN patients. Our tasks were to investigate the percentage of the patients' neutrophils that express $\mathrm{CD} 11 \mathrm{~b} / \mathrm{CD} 18$ and compare it with the control group, to define the type of MPN that has the highest expression of the marker, and also find an association between granulocytic CD11b/CD18 expression of MPN patients and a history of thrombotic complication.

\section{Materials/Patients and Methods}

We selected 110 patients with a confirmed diagnosis of MPN (according to WHO 2008 and WHO 2016 criteria and classification), aged 2390 years (median age 62.87 years), hospitalized at the Hematology Clinic of the Georgi Stranski University Hospital or on an out-patient setting in Pleven, Bulgaria between March 8, 2013, and July 1, 2020.

We compared CD11b/CD18 expression by flow cytometry, investigating a control group of 46 healthy volunteers with no history of MPN or thrombotic complication (average age 62.52 years, female: male ratio $=1: 2$ ).

Informed consent forms and questionnaires were obtained from all the participants. The questionnaire included information about passport data, disease history and ongoing therapy, concomitant medications, and other medical conditions (myocardial infarction, ischemic heart disease, arterial hypertension, heart failure, diabetes, obesity, hyperlipidemia, liver dysfunction, or solid tumors). We focused on past thrombotic events before and after diagnosis, family history of thrombotic complications, risk factors for thrombogenesis - recent surgery, trauma, prolonged immobilization, neoplasms, hormone therapy, and smoking. In female patients, special attention was paid to a history of spontaneous abortion and pregnancy complications.
Venous blood $(7-10 \mathrm{ml})$ was obtained from all patients. We used flow cytometric analysis to determine the $\mathrm{CD} 11 \mathrm{~b} / \mathrm{CD} 18$ expression in percentage. White blood cells were investigated 2 hours after venous blood was obtained, using a dual-laser FACSCalibur cytometer (Becton Dickinson, Heidelberg, Germany). The analysis was performed with Cell Quest computer software. Blood cells were processed with two monoclonal antibodies, marked with two fluorochrome stains. After erythrocyte lysis (lysis buffer; Becton Dickinson) and two-step washing, white blood cells bound to monoclonal antibodies were resuspended and fixed in formaldehyde solution. After obtaining 10000 cells from each patient, we investigated the cell size and granularity, using forward and side scatter (FSC/SSC) to define the population of interest (gating). The flow cytometer was calibrated daily with calibration beads, and results were analyzed with FACS Comp software@2007 Becton Dickinson. Cell subpopulations were identified by monoclonal antibodies fluorescence CD11b FITC, CD18 PE.

\section{Results}

Sex distribution was 47 females and 63 males - 1:1.34. Of all 110 patients: 34 (30.91\%) were diagnosed with PV, 13 (11.82\%) - with ET, 35 $(31.82 \%)$ - with MF, and $28(25.45 \%)$ - with CML. (Figure 1)

Median CD11b/CD18 granulocytic expression for all patients was $70.64 \%$. Expression was $15.38 \%$ for the control group, i.e., 4.59 times lower. (Figure 2)

Median CD11b/CD18 expression was highest in the PV subgroup of patients $-71.55 \%$ of their neutrophils expressed the markers, i.e., 4.65 times more than that in the controls. Nine of these patients $(26.47 \%$ of the $34 \mathrm{PV}$ patients) had a medical history of thrombosis. The expression was $69.07 \%$ - lower than the median PV subgroup expression, but 4.49 times higher than in the control group. (Figure 3)

In the ET subgroup, $69.48 \%$ of the patients' neutrophils expressed CD11b/CD18 (4.52 times more than in the control group). Five of these patients (38.46\% of 13 patients) had a medical history of thrombosis, and their expression was $81.11 \%$ - higher than the median ET subgroup 


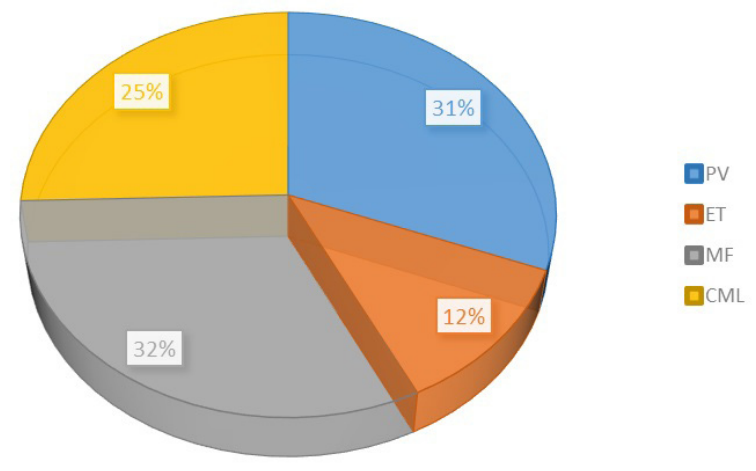

Figure 1. Patient distribution according to diagnosis

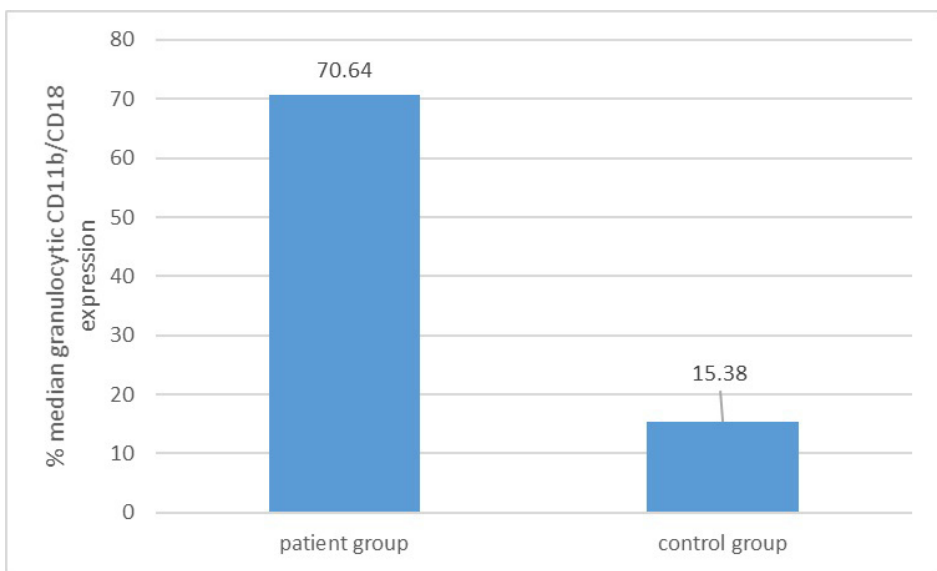

Figure 2. Median granulocytic CD11b/CD18 expression in patient and control group

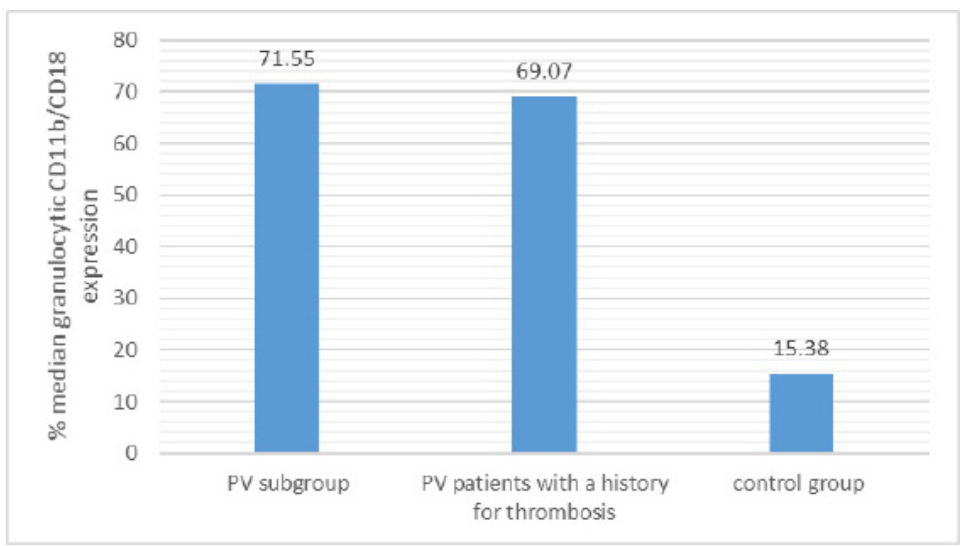

Figure 3. Median granulocytic CD11b/CD18 expression in PV patients subgroup and control group

expression and 5.27 times that of the control group. (Figure 4)

In the MF subgroup, $68.47 \%$ of patients' neutrophils expressed CD11b/CD18 (4.45 times more than in the control group). Eleven of these patients (31.43\% of 35 patients) had a medical history of thrombosis, and their expression was $72.21 \%$ - higher than the median MF subgroup expression and 4.7 times more than in the controls. (Figure 5)

In the CML subgroup, $65.56 \%$ of the patients' neutrophils expressed CD11b/CD18 (4.26 times more than in the control group). Five of these patients $(17.86 \%$ of 28 patients) had a medical history for thrombosis, and their expression was 65.91\% - higher than the median CML subgroup expression and 4.29 times more than in the control group. (Figure 6) 


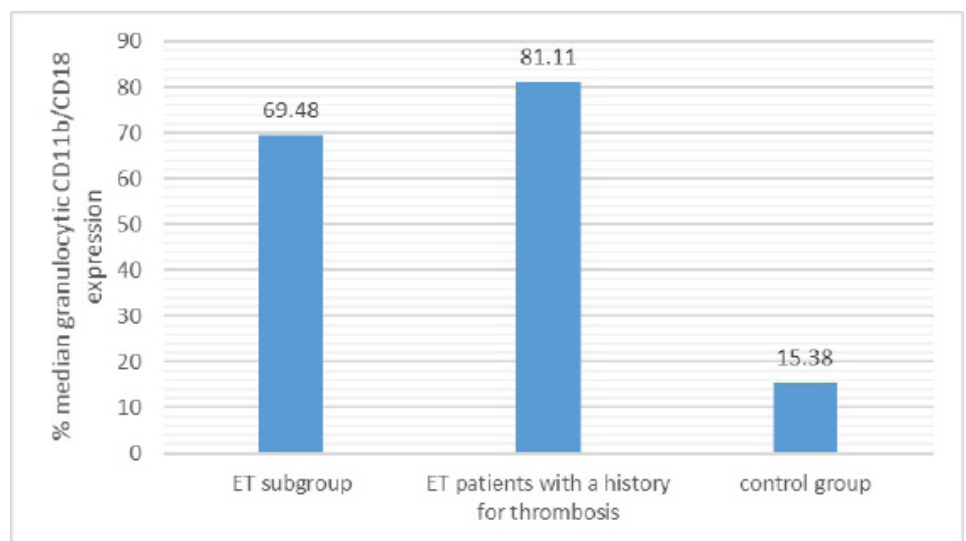

Figure 4. Median granulocytic CD11b/CD18 expression in ET patients subgroup and control group

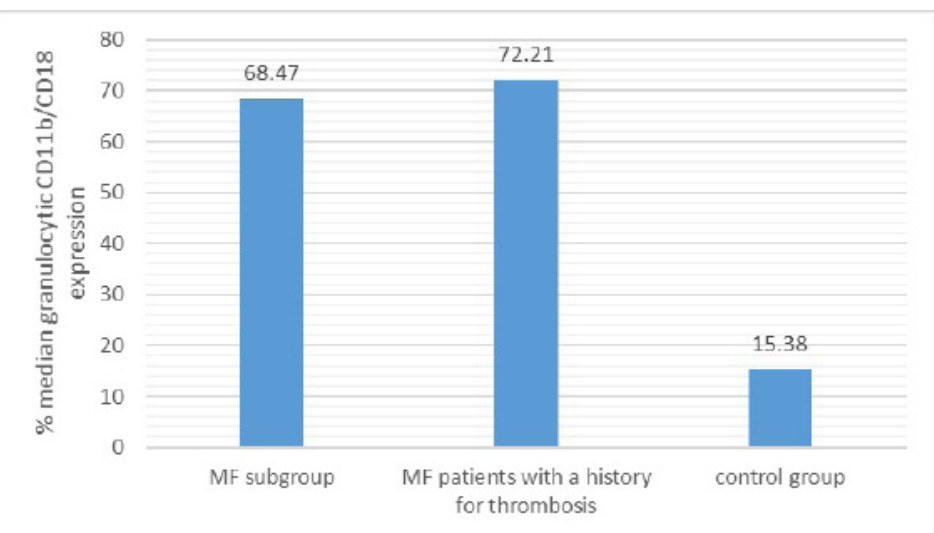

Figure 5. Median granulocytic CD11b/CD18 expression in MF patients subgroup and control group

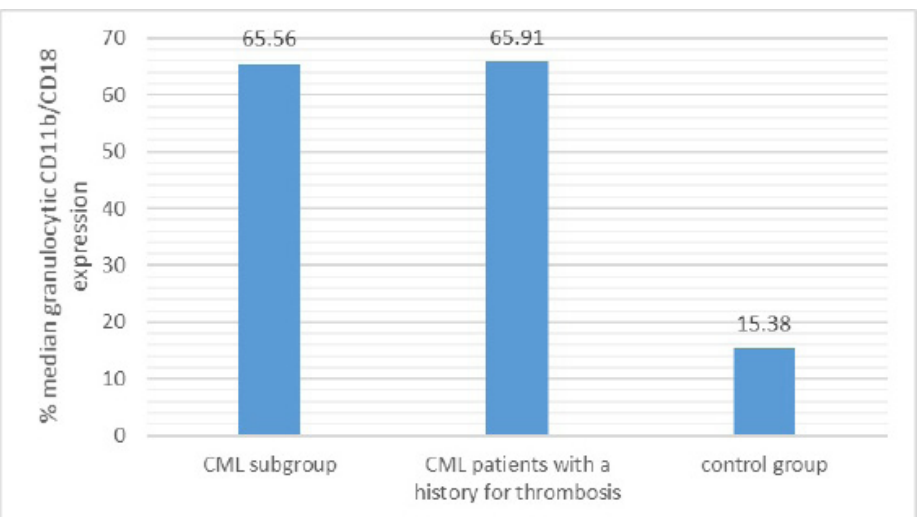

Figure 6. Median granulocytic CD11b/CD18 expression in CML patients subgroup and control group

\section{Discussion}

Thrombotic complications in MPN patients are frequent. One possible cause for this is formation of leucocyte-platelet aggregates [16]. Flow cytometry can confirm the presence of platelet microparticles and aggregates (anti-CD62p) and Mac-1 expression (CD11b/CD18), contributing to platelet and leukocyte activation, respectively. We found that MPN patients' neutrophils expressed a 4.59 times higher percentage of
CD11b/CD18 than that in the controls. Most investigators focus on PV and ET and not on the MPN group as a whole [18]. They have confirmed the role of activated neutrophils in the pathogenesis of thrombosis in these patients. We also found that the highest number of neutrophils expressing CD11b/CD18 was in the PV and ET subgroups. However, the highest number of patients with a history of thrombosis was in the ET subgroup (38.46\% of all ET patients), and their median expression was the highest we 
found $-81.11 \%$. This result may also be related to thrombocytosis, which is typical for this disease. Testing for platelet activation markers would be relevant (CD61, CD41, CD42b) in defining a possible relationship with our results. A higher percentage of neutrophils expressed CD11b/CD18 in patients who had experienced thrombosis than those without such history for ET, MF, and CML subgroups. These data suggest that testing for leucocyte activation markers should be performed to define thrombotic risk for the whole MPN group.

\section{Conclusions}

The overall median CD11b/CD18 neutrophilic expression for the MPN group was 4.6 times higher than in the control group. The highest percentage of neutrophils expressed these markers in the PV subgroup. In the ET subgroup, the highest number of patients experienced thrombotic complications with the highest number of neutrophils expressing CD11b/CD18. The neutrophils' CD11b/CD18 expression was higher in ET, MF, and CML patients with a history of thrombosis than the patients in these groups without such a history.

\section{Acknowledgments}

Study data are results of scientific projects No 13/2013, No 2/2015, No 9/ 2017, and N9/ 2019, financed by Medical University - Pleven, Bulgaria.

The authors would like to express their gratitude to Medical University - Pleven, Bulgaria.

\section{References}

1. Gamal TE, Mohamed G, Omina S, Mahmoud MK. Prevalence of the frequency of JAK2 (V617F) mutation in different myeloproliferative disorders in Egyptian patients. Int J Clin Exp Pathol. 2015;8(9):11555-9.

2. Da Silva RR, Hatzlhofer BLD, Machado CGF, Lima ASM, Albuquerque DM, Santos MNN et al. JAK2 V617F mutation prevalence in myeloproliferative neoplasms in Pernambuco, Brazil. Genet Test Mol Biomarkers. 2012;16(7):802-5.

3. Azevedo A, Silva SN, Reichert A, Lima F,
Júnior E., Rueff J. Prevalence of the Janus kinase 2 V617F mutation in Philadelphianegative myeloproliferative neoplasms in a Portuguese population. J. Biomedical Reports. 2017;7(4):370-6.

4. Tefferi A, Pardanani A. Myeloproliferative Neoplasms: A Contemporary Review. JAMA Oncol. 2015;1(1):97-105.

5. Levine RL. Mechanisms of mutations in myeloproliferative neoplasms. Best Pract Res Clin Haematol. 2009;22(4):489-94.

6. Passamonti F, Rumi E, Pietra D, Della Porta MG, Boveri E, Pascutto C et al. Relation between JAK2 (V617F) mutation status, granulocyte activation, and constitutive mobilization of CD34+ cells into peripheral blood in myeloproliferative disorders. Blood. 2006;107(9):3676-82.

7. Singdong R, Siriboonpiputtana T, Chareonsirisuthigul $\mathrm{T}, \quad$ Kongruang A, Limsuwanachot N, Sirirat $\mathrm{T}$ et al. Characterization and Prognosis Significance of JAK2 (V617F), MPL, and CALR Mutations in Philadelphia-Negative Myeloproliferative Neoplasms. Asian Pacific journal of cancer prevention: APJCP. 2016;17(10):4647-53.

8. Kralovics R, Passamonti F, Buser AS, Teo SS, Tiedt R, Passweget JR et al. A gain-of-function mutation of JAK2 in myeloproliferative disorders. N Engl J Med. 2005;352(17):177990.

9. Passamonti F, Maffioli M, Caramazza D, Cazzola M. Myeloproliferative neoplasms: from JAK2 mutations discovery to JAK2 inhibitor therapies. Oncotarget. 2011;2(6):485-90.

10. Barbui T, Finazzi G, Falanga A; Myeloproliferative neoplasms and thrombosis. Blood. 2013;122(13):2176-84.

11. Barbui T, Carobbio A, Cervantes F, Vannucchi AM, Guglielmelli P, Antonioli $\mathrm{E}$ et al. Thrombosis in primary myelofibrosis: incidence and risk factors. Blood. 2010;115(4):778-82.

12. Elliott MA, Tefferi A. Thrombosis and haemorrhage in polycythaemia vera and essential thrombocythaemia. Br J Haematol. 2005;128(3):275-90.

13. Passamonti F, Rumi E, Arcaini L, Boveri E, Elena C, Pietra D et al. Prognostic factors for thrombosis, myelofibrosis, and leukemia in essential thrombocythemia: a study of 605 patients. Haematologica. 2008;93(11):1645-51.

14. Carobbio A, Finazzi G, Guerini V, Spinelli O, Delaini F, Marchiolie R et al. Leukocytosis is a risk factor for thrombosis in essential thrombocythemia: interaction with treatment, standard risk factors, and Jak2 mutation status. Blood. 2007;109(6):2310-3. 
15. Falanga A, Marchetti M, Barbui T, Smith CW. Pathogenesis of thrombosis in essential thrombocythemia and polycythemia vera: the role of neutrophils. Semin Hematol. 2005;42(4):239-47.

16. Jensen MK, de Nully Brown P, Lund BV, Nielsen OJ, Hasselbalch HC. Increased circulating platelet-leukocyte aggregates in myeloproliferative disorders is correlated to previous thrombosis, platelet activation and platelet count. Eur J Haematol. 2001;66(3):14351.
17. Alvarez-Larrán A, García-Pagán JC, Abraldes JG, Arellano E, Reverter JC, Bosch J et al. Increased CD11b neutrophil expression in Budd-Chiari syndrome or portal vein thrombosis secondary to polycythaemia vera. Br J Haematol. 2004;124(3):329-35.

18. Falanga A, Marchetti M, Evangelista V, Vignoli A, Licini M, Balicco M et al. Polymorphonuclear leukocyte activation and hemostasis in patients with essential thrombocythemia and polycythemia vera. Blood. 2000;96(13):4261-6. 